\title{
APERTURE SYNTHESIS CS $(J=1-0)$ OBSERVATIONS OF THE MOLECULAR OUTFLOW IN NGC 2071: SUBCLUMPS IN A WIND-DRIVEN SHOCKED SHELL AND THEIR ORIGIN
}

\author{
Yoshimi KITAMURA \\ School of Allied Medical Sciences, Kagoshima University, Sakuragaoka 8-35-1, \\ Kagoshima 890, Japan

\begin{abstract}
Ryohei KAWABE, and Masato ISHIGURO
Nobeyama Radio Observatory, Nobeyama, Minamimaki-mura, Minamisaku-gun, Nagano 384-13, Japan
\end{abstract}

\begin{abstract}
Aperture synthesis CS(J=1-0) observations were made of the expanding clumpy shocked shell that was found around the redshifted $C O$ outflow in NGC 2071 during our previous study (Kitamura et al. 1990). It has been revealed that previously identified clumps in the shell comprise several small subclumps. The decelerating shock and Rayleigh-Taylor instabilities seem to be probable mechanisms by which the subclumps are formed in the shock-compressed shell.
\end{abstract}

Source NGC 2071 is one of the best objects for detailed investigation of clumpy structure at the interface between the outflow and ambient clouds: Our previous study (Kitamura et al. 1990) found the expanding clumpy shocked shell around the redshifted CO outflow and identified several clumps, which can be resolved by small beams of interferometers. Therefore, we have made aperture synthesis observations of the shocked shell by using Nobeyama Millimeter Array from 1988 April to 1990 March (Kitamura, Kawabe, and Ishiguro 1992). The size of the synthesized beam was $\sim 11 " \times 10^{\prime \prime}(0.02 \mathrm{pc} \times 0.02 \mathrm{pc})$ and the velocity resolution was $1.91 \mathrm{~km} \cdot \mathrm{s}^{-1}$.

It has been revealed that previously identified clumps in the shocked shell comprise several small subclumps. We have identified 22 subclumps, according to the following criteria: (1) each subclump must have one or more local peaks higher than the $6 \sigma$ level in each channel map, (2) in the sky plane, the distance between the peaks of two neighboring subclumps must be greater than the beam size of $\sim 10 "$, or (3) the difference between the velocities of two subclumps must be greater than the velocity resolution of $1.91 \mathrm{~km} \cdot \mathrm{s}^{-1}$. Figure 1 shows the distribution functions of the size, velocity width (full width at zero intensity), and mass of the subclumps. The central velocities of the subclumps seem to be randomly distributed over a velocity range of $V_{L S R}=5$ to $22 \mathrm{~km} \cdot \mathrm{s}^{-1}$. Figure 2 shows the relationships among the physical properties of the subclumps.

To discuss the origin of the subclumps, we consider the following six possibilities: 1) decelerating shock instability; 2) Rayleigh-Taylor instability; 3) Kelvin-Helmholtz instability; 4) thermal instability; 5) Jeans instability; and 6) a pre-existing inhomogeneity. All but the first possibility have been discussed in our paper (Kitamura, Kawabe, and Ishiguro 1992) and it was concluded that the Rayleigh-Taylor instability seems to be a probable process to produce clumpiness in the shocked shell. Therefore, we discuss the decelerating shock instability in this study.

Shock waves induced by molecular outflows around protostars are usually decelerating owing to sweeping up of the ambient gas. In such decelerating shock waves, instability occurs and the growth rate of perturbations takes a maximum at a finite wavelength (Ryu and Vishniac 1988; Vishniac and Ryu 1989). Based on the instability, Elmegreen and Lada (1977) proposed a scenario of sequential star formation in Orion. In the case of NGC 2071, the instability of the decelerating shock would produce the 
subclumps found by us. Using a linear perturbation theory, Nishi (1992) derived the wavelength $\lambda$ and mass $M$ that correspond to the maximum growth rate of perturbations in the decelerating shock:

$$
\lambda \approx 0.02 \mathrm{pc}\left(\frac{n}{10^{5} \mathrm{~cm}^{-3}}\right)^{-0.5}\left(\frac{M a c h}{12}\right)^{-1}\left(\frac{T}{30 \mathrm{~K}}\right)^{0.5} \quad M \approx 5.5 M_{\odot}\left(\frac{M a c h}{12}\right)^{-1}\left(\frac{T}{30 \mathrm{~K}}\right)^{1.5}
$$

Here $n, T$, and Mach mean the density and temperature of the compressed gas, and the Mach number corresponding to the shock velocity. Self-gravity is taken into account in these equations. This mode corresponds to Elmegreen's E-mode (Elmegreen 1989), a collapse after one oscillation. It is noted that the wavelength and mass in the above equations agree well with the size and mass of the subclumps found by us (see Fig. 1). Since all the subclumps seem to be gravitationally unbound (see the right panel of Fig. 2 ), it is suggested that the gravitational instability does not dominate the decelerating shock instability. If we take a high value of $10^{6} \mathrm{~cm}^{-3}$ for the gas density, the wavelength $\lambda$ becomes smaller than $0.01 \mathrm{pc}$. Actually, our observations suggest the existence of unresolved subclumps smaller than $0.01 \mathrm{pc}$ (see the left panel of Fig. 1). Of course, the range of the wavelength depends on the fluctuation of the Mach number. In contrast with the wavelength, the mass $M$ depends very weakly on the gas density, and the wide range of the subclump mass, shown in the right panel of Fig. 1, is probably attributed to the fluctuation of the temperature of the compressed gas.
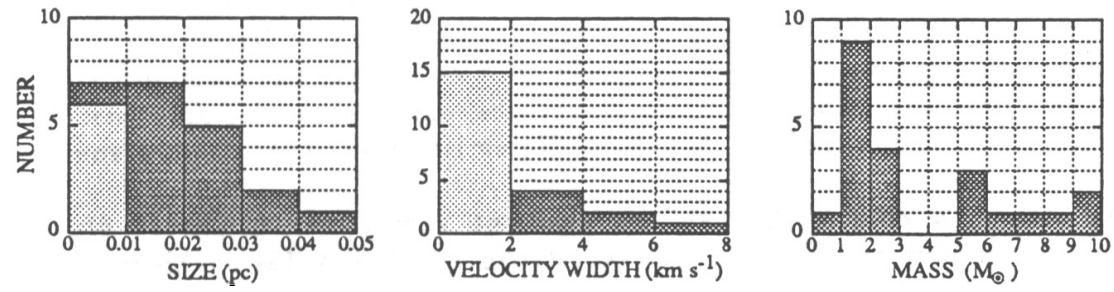

Figure 1: Distribution functions of the size $(\mathrm{pc})$, velocity width $\left(\mathrm{km} \cdot \mathrm{s}^{-1}\right)$, and mass $\left(M_{\odot}\right)$ of the identified subclumps. In the left and middle panels, the lightly shaded bars on the left side indicate the subclumps whose sizes or velocity widths could not be resolved by this observation.
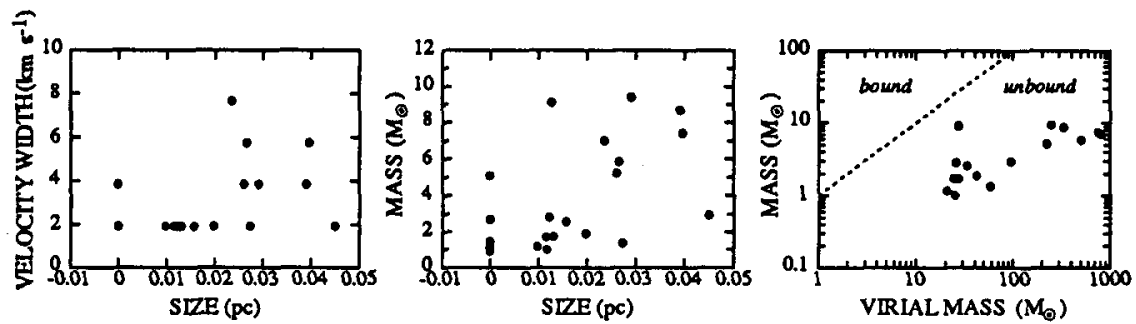

Figure 2: Relationships among the physical properties of the subclumps; the size (pc), velocity width $\left(\mathrm{km} \cdot \mathrm{s}^{-1}\right)$, mass $\left(M_{\odot}\right)$, and virial mass $\left(M_{\odot}\right)$.

\section{REFERENCES}

Elmegreen 1989, Ap. J., 340, 786

Elmegreen and Lada 1977, Ap.J., 214, 725

Kitamura, Kawabe, and Ishiguro 1992, Publ. Astron. Soc. Japan, 44, 407

Kitamura et al. 1990, Ap.J., 363, 180

Nishi 1992, Prog. Theor. Phys., 87, 347

Ryu and Vishniac 1988, Ap.J., 331, 350

Vishniac and Ryu 1989, Ap.J., 337, 917 